\title{
A delay-minimizing routing strategy for wireless multi-hop networks
}

\author{
Kostas Stamatiou*, Francesco Rossetto ${ }^{\dagger}$, Martin Haenggi ${ }^{\ddagger}$, Tara Javidi*, James R. Zeidler* and Michele Zorzi ${ }^{\S}$ \\ *University of California San Diego, La Jolla, CA 92037 \\ ${ }^{\dagger}$ DLR, Institute of Communications and Navigation, 82234 Weßling, Munich, Germany \\ †University of Notre Dame, Notre Dame, IN 46556

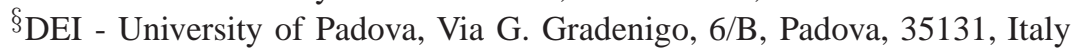 \\ Email:kostas@ucsd.edu, franzross@gmail.com
}

\begin{abstract}
We consider a network where each route comprises a backlogged source, a number of relays and a destination at a finite distance. The locations of the sources and the relays are realizations of independent Poisson point processes. Given that the nodes observe a TDMA/ALOHA MAC protocol, our objective is to determine the number of relays and their placement such that the mean end-to-end delay in a typical route of the network is minimized. We first study an idealistic network model where all routes have the same number of hops, the same distance per hop and their own dedicated relays. Combining tools from queueing theory and stochastic geometry, we provide a precise characterization of the mean end-to-end delay. We find that the delay is minimized if the first hop is much longer than the remaining hops and that the optimal number of hops scales sublinearly with the source-destination distance. Simulating the original network scenario reveals that the analytical results are accurate, provided that the density of the relay process is sufficiently large. We conclude that, given the considered MAC protocol, our analysis provides a delay-minimizing routing strategy for random, multihop networks involving a small number of hops.
\end{abstract}

\section{INTRODUCTION}

The premise of multi-hop transmission is that, for a given power, a larger received signal-to-interference-and-noise-ratio can be achieved on a per hop basis, compared to transmitting directly to the final destination. This implies a higher packet success probability and/or information rate per hop. However, as argued in [1], a large number of short hops does not necessarily result in a smaller end-to-end packet delay. On the contrary, each node that is added between the source and the destination is also the cause of additional delay since a packet has to be decoded, re-encoded and wait in the queue before it is successfully transmitted to the next node.

The purpose of this paper is to conduct a systematic study of the end-to-end delay performance in a wireless network, taking into account the fading and interference associated with wireless transmissions. We consider a network that consists of a population of backlogged sources, each with a destination at a finite distance $R$, and a population of relays, that are employed to forward the source packets to their respective destinations. The locations of the sources and the relays are randomly drawn according to independent Poisson point processes. For the sake

This work was supported by the MURI Grant W911NF-04-1-0224 and the DARPA IT-MANET Grant W911NF-07-1-0028. Francesco Rossetto was partially supported by the "Ing. Aldo Gini" Foundation, Padova, Italy. of analysis, we initially simplify this model and assume that each route has the same number of hops, the same distance per hop and its own dedicated relays. Given that the nodes observe a TDMA/ALOHA MAC protocol, we use well established tools from stochastic geometry and queueing theory, in order to evaluate the mean end-to-end delay and throughput in a typical route. Our analytical findings are then tested by simulating the original network setting.

\section{A. Previous work}

This work bridges the gap between two existing research directions. In the first one [2]-[5], a single hop of a multihop route is studied, under the implicit assumption that the destination lies somewhere at an infinite distance. Singlehop metrics are devised and evaluated such as the expected progress [2], [6], i.e., the product (packet success probability) $\times$ (hop length), defined for a given spatial density of transmitters, which reflects the reliability/forward-progress tradeoff; and the transmission capacity [3], [7], i.e., the maximum allowable network throughput, defined for a given hop length, such that a constraint on the packet success probability is satisfied. In the second body of work [8]-[10], a well defined route is considered, where the distance to the final destination and the number of relays are specified. However, the impact of interference from other transmissions in the network is not taken into account. A step in the study of random multi-hop networks is made in [11], where the benefits of opportunistic vs. shortest-path routing are evaluated.

\section{B. Contributions}

Assuming that the network reaches a stationary regime, we derive closed-form expressions for the mean end-to-end delay and throughput in a typical route of the network. It is found that the delay is minimized if the first hop is much larger than the remaining hops, e.g., in a delay-optimized three hop route, the first hop can cover half the total distance. Moreover, we demonstrate that the optimal number of hops scales sublinearly with the source-destination distance. Simulations are performed with the original network model, where the relays of each route are selected out of a random population, according to the above guidelines. The simulation confirms the validity of the analysis for a sufficiently large relay density and 
TABLE I: Commonly used symbols

\begin{tabular}{|c|c|}
\hline Symbol & Meaning \\
\hline \hline$p$ & source and relay transmission probability \\
\hline$\lambda$ & density of sources \\
\hline$\lambda_{r}$ & density of relays \\
\hline$R$ & source-destination distance \\
\hline$N$ & number of hops \\
\hline$p_{n}^{s}$ & success probability over hop $n$ \\
\hline$r_{n}$ & distance of hop $n$ \\
\hline
\end{tabular}

that departure from the design guidelines leads to significant delay penalties.

\section{SYSTEM MODEL}

\section{A. Network setting}

We consider a network composed of an infinite number of backlogged sources, each with a destination at a finite distance $R$ and a random orientation, and an infinite population of relays. The locations of the sources and the relays are drawn independently according to spatially homogeneous Poisson point processes of densities $\lambda$ and $\lambda_{r}$, respectively. A route in the network consists of a source, a finite number of relays that forward the source packets, and a final destination (see Fig.1).

Each node has an infinite queue, where packets that are received from the previous node in the route can be stored in a first-in, first-out fashion. Time is divided into packet slots. Within a route, a TDMA/ALOHA protocol is observed, according to which a node is allowed to transmit every $N$ slots with probability $p$. A packet is received successfully by a node, if the SIR in that slot is above a target threshold $\theta$. If it is not, the transmitting node is informed via an ideal feedback channel and the packet remains at the head of its queue until the node gets another opportunity to transmit. Note that this MAC protocol is tailored to routes with a relatively small number of hops, as it results in no intra-route interference [10].

The network operation starts at some arbitrary time, with an arbitrary number of packets in each queue. For convenience, we assume that different routes are synchronized at the slot level and that the node of each route which is first allowed to transmit is selected with probability $1 / N$. Since the population of relays is common, there is a possibility that, at a given slot, a relay has to be used, either for transmission or reception, by more than one routes. We assume that the conflict is resolved arbitrarily.

The analysis of the network model described above is complicated by the fact that the number of relays and their placement differ across routes, as well as the fact that routes might intersect. In order to simplify the model, we let each route in the network have the same number of hops $N$, the same distance per hop $r_{n}, n=1, \ldots, N$, and its own set of relays. The design guidelines that stem from the analysis of the idealized model are then used to route packets in a simulation of the original realistic network.

\section{B. Physical layer}

The channel between two nodes at distance $r$ includes Rayleigh fading - with a coherence time of one slot - and path-

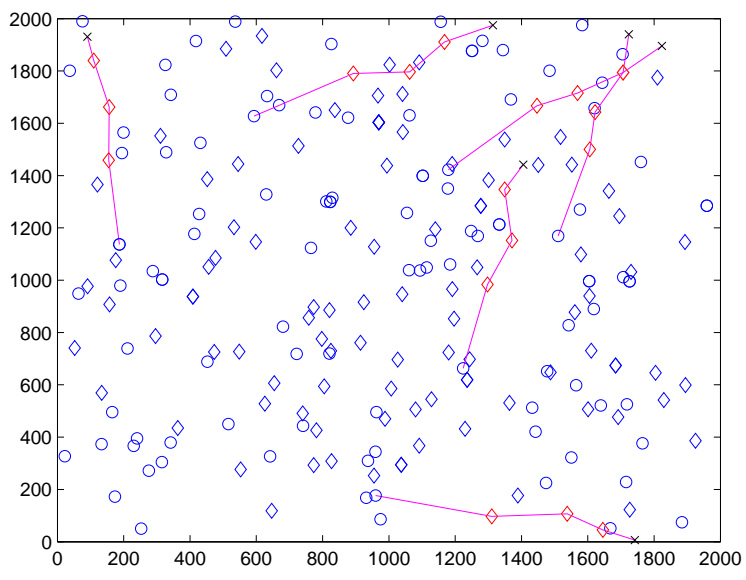

Fig. 1: A network realization $\left(\lambda=10^{-4}\right.$ sources $/ \mathrm{m}^{2}, \lambda_{r}=3 \lambda$, $R=800 \mathrm{~m}$ and $N=4$ ). Sources are denoted by circles, relays by diamonds and destinations by x's. Only $10 \%$ of the nodes and a few routes are shown in order to avoid cluttering the figure.

loss according to the law $r^{-b}$, where $b>2$ is the path-loss exponent. We consider an interference-limited setting, where thermal noise is assumed to be negligible and all nodes have the same transmit power, which is normalized to one. The signal-to-interference-ratio (SIR) at the $n^{\text {th }}$ hop of a typical route is therefore

$$
\operatorname{SIR}_{n}=\frac{A_{n} r_{n}^{-b}}{\sum_{i \in \Phi_{n}} B_{i} d_{i}^{-b}}, n=1, \ldots, N
$$

where $r_{n}$ is the distance of the $n^{\text {th }}$ hop; $A_{n}$ is the fading coefficient between the transmitting and receiving node and exponentially distributed with unit mean; $\Phi_{n}$ is the process of interfering nodes over hop $n ; d_{i}$ is the distance between interfering node $i$ and the receiver and $B_{i}$ is the respective fading coefficient, also exponentially distributed with unit mean.

The probability of successful packet reception at the $n^{\text {th }}$ hop is defined as $p_{n}^{s}=\mathbb{P}\left(\operatorname{SIR}_{n}>\theta\right)$. The following result provides an expression for $p_{n}^{s}$, when $\Phi_{n}$ is a homogeneous Poisson point process.

Lemma 1 (Corollary 3.2 [5]): Given the definition of $\mathrm{SIR}_{n}$ in (1) and that $\Phi_{n}$ is a homogeneous Poisson point process with density $\lambda_{n}$

$$
p_{n}^{s}=\mathrm{e}^{-\lambda_{n} c r_{n}^{2}},
$$

where $c=\Gamma(1+2 / b) \Gamma(1-2 / b) \pi \theta^{2 / b}$ and $\Gamma(x), x>0$, is the gamma function.

\section{Network metrics}

The metric of interest is the mean end-to-end delay $D$, i.e., the mean total time (in slots) that it takes a packet to travel from the source to the destination in a typical route. Assuming negligible propagation times, $D$ is the sum of the mean waiting times and service times along the queues of the route. The 
waiting time at a given node is measured starting from the moment a packet arrives at that node's queue, till it becomes the head-of-line packet, i.e., all packets in front of it have been successfully transmitted to the next node. The service time is measured from the moment a packet reaches the head of the queue, till it is successfully received by the next node and includes the access delay associated with the MAC protocol.

In addition to the delay, a metric of interest is the Route Throughput (RT), defined as the expected number of packets successfully delivered to the destination per slot. The respective Network Throughput (NT) is then defined as NT $=\lambda \mathrm{RT}$.

\section{ANALYSIS}

To begin our analysis, we assume that, under certain conditions which will be discussed in Section III-C, the queues approach a stationary behavior in the long run. Moreover, due to the randomness and symmetry present in our network model, we assume that, in this stationary regime, successful packet transmissions over the $n^{\text {th }}$ hop of the typical route are independent events that occur with probability $p_{n}^{s}$. The latter assumption allows us to use (2) in order to derive a set of fixed-point equations that provide solutions for the success probabilities $\left\{p_{n}^{s}\right\}_{n=1}^{N}$. Our approach is reminiscent of [12], where a decoupling approximation was made in order to analyze the performance of the IEEE 802.11 protocol for wireless local area networks.

In the first part of this section, we use tools from queueing theory in order to express the mean delay as a function of $p$ and $\left\{p_{n}^{s}\right\}_{n=1}^{N}$. In the second part, we evaluate $\left\{p_{n}^{s}\right\}_{n=1}^{N}$.

\section{A. Queueing analysis}

Assume that we are looking at the queue of the first relay. A packet arrival occurs at the end of slot $m N, m \in \mathbb{Z}$, if the source transmitted in that slot and the transmission was successful. The probability of this happening is $p_{I}=p p_{1}^{s}$. Provided that the queue is not empty, at the beginning of slot $1+m N$, a packet departs from the head of the queue with probability $p_{O}=p p_{2}^{s}$. This procedure is repeated every $N$ slots.

Modeling the queue of the relay as a Random Walk, whose state is the number of packets in the queue at the end of slot $m N$, we can derive the steady-state probability of being in state $k$ [13]. The mean waiting time at the first relay, $Q_{1}$, can then be computed by Little's theorem, as the average queue size, excluding the head-of-line packet, divided by the arrival rate, in this case $p_{I} / N$. The resulting expression is

$$
Q_{1}=N \frac{p_{I}}{p_{O}} \frac{1-p_{O}}{p_{O}-p_{I}} .
$$

It is also straightforward to show that the service time for the head-of-line packet is

$$
H_{1}=N\left(\frac{1}{p_{O}}-1\right)+1,
$$

so the total time in the queue of the first relay is

$$
\begin{aligned}
Q_{1}+H_{1} & =N \frac{p_{I}}{p_{O}} \frac{1-p_{O}}{p_{O}-p_{I}}+\frac{N}{p_{O}}-N+1 \\
& =N \frac{1-p_{I}}{p_{O}-p_{I}}-N+1 .
\end{aligned}
$$

If the queue of the first relay is stable, i.e., $p_{1}^{s}<p_{2}^{s}$, the packet arrival probability to the second relay is $p p_{1}^{s}$. In fact, as long as all intermediate queues are stable, i.e., $p_{1}^{s}<p_{n}^{s}$, $n=2, \ldots, N$, the packet arrival probability to relay $n$ is $p p_{1}^{s}$. We can thus calculate the total time in the queue of relay $n$ by setting $p_{I}=p p_{1}^{s}$ and $p_{O}=p p_{n}^{s}$ in (3).

Adding the service time at the source and the total times at the queues of $N-1$ relays, the mean end-to-end delay for a packet at the head of the source queue, $D$, is found to be ${ }^{1}$

$$
D=\frac{N}{p p_{1}^{s}}+N \sum_{n=2}^{N} \frac{1-p p_{n}^{s}}{p p_{n}^{s}-p p_{1}^{s}} .
$$

Since a packet is received by the destination every $N$ slots with probability $p p_{1}^{s}$, the route throughput is $\mathrm{RT}=p p_{1}^{s} / N$.

\section{B. Evaluation of packet success probabilities}

The following facts are useful and are stated without proof.

Fact 1: The Lambert function, $y=W(x)$, is the unique solution to the equation $y \mathrm{e}^{y}=x, x \geq-\mathrm{e}^{-1}$ and $y \geq-1$.

Fact 2: The function $g(x)=x \mathrm{e}^{\mu x}, \mu>0, x \geq 0$, is continuous and strictly increasing. The equation $y=g(x), \mu>$ $0, x, y \geq 0$, has a unique solution $x_{1}=W(\mu y) / \mu$.

Under the assumption that packet success events are independent across routes, the queue of relay $n$ of the typical route is not empty at the beginning of its assigned slot with probability $p_{1}^{s} / p_{n}^{s}$, hence the relay transmits a packet with probability $p p_{1}^{s} / p_{n}^{s}$. As a result, the interfering nodes over hop $n$ constitute a homogeneous Poisson point process with density

$$
\lambda_{n}=\frac{\lambda p}{N}+\frac{\lambda p}{N} \sum_{k=2}^{N} \frac{p_{1}^{s}}{p_{k}^{s}},
$$

where the first term represents the transmitting sources, while the second term represents the total population of transmitting relays. From Lemma 1, $p_{n}^{s}$ satisfies the fixed-point equation

$$
p_{n}^{s}=\mathrm{e}^{-\frac{\lambda}{N} p\left(1+\sum_{k=2}^{N} \frac{p_{1}^{s}}{p_{k}^{s}}\right) c r_{n}^{2}}, n=1, \ldots, N .
$$

Eq. (5) illustrates the coupling effect between concurrent transmissions in a wireless network. The packet success probabilities depend on the interference level in the network and vice versa.

The solution of (5) over $\left\{p_{n}^{s}\right\}_{n=1}^{N}$ for general values of $r_{1}, \ldots, r_{N}$ appears complicated. However, if $r_{2}=\cdots=r_{N}$, i.e., hops $2, \ldots, N$ are equidistant, we have $p_{2}^{s}=\cdots=p_{N}^{s}$ and (5) results in

$$
p_{n}^{s}=\mathrm{e}^{-\frac{\lambda}{N} p\left(1+(N-1) \frac{p_{1}^{s}}{p_{n}^{s}}\right) c r_{n}^{2}}, n=1,2 .
$$

\footnotetext{
${ }^{1}$ Since the sources are backlogged, the only meaningful way to define the end-to-end delay is for a packet at the head of the source queue.
} 
Dividing the two expressions and arranging terms

$$
\frac{p_{1}^{s}}{p_{2}^{s}} \mathrm{e}^{\frac{\lambda}{N}(N-1) p c\left(r_{1}^{2}-r_{2}^{2}\right) \frac{p_{1}^{s}}{p_{2}^{s}}}=\mathrm{e}^{-\frac{\lambda}{N} p c\left(r_{1}^{2}-r_{2}^{2}\right)} .
$$

By Fact 2, (7) has the following unique solution with respect to $p_{1}^{s} / p_{2}^{s}$

$$
\frac{p_{1}^{s}}{p_{2}^{s}}=\frac{W\left(\frac{\lambda}{N}(N-1) p c\left(r_{1}^{2}-r_{2}^{2}\right) \mathrm{e}^{-\frac{\lambda}{N} p c\left(r_{1}^{2}-r_{2}^{2}\right)}\right)}{\frac{\lambda}{N}(N-1) p c\left(r_{1}^{2}-r_{2}^{2}\right)} .
$$

Substituting (8) in (6), the solutions for $p_{1}^{s}, p_{2}^{s}$ can be obtained.

The following proposition is quite straightforward to prove and shows that setting $r_{2}=\cdots=r_{N}$ minimizes $D$.

Proposition 1: The mean delay, given by (4), is minimized when the relays are placed on the line between the source and the destination and $r_{2}=\cdots=r_{N}=\frac{R-r_{1}}{N-1}$.

Proof: See [13].

\section{A discussion on stability}

In the preceding subsections, we evaluated the mean endto-end delay over a typical route, based on the assumptions that the queues approach a stationary behavior in the long run and that packet successes are independent across routes. The latter assumption is justified when the probability of random access $p$ is sufficiently small [14].

Determining if and under what conditions the network attains a stationary regime, i.e., it is dynamically stable [5], is a complicated problem, as transmissions interfere with each other, creating couplings between different queues. However, we can determine conditions under which the network is rate stable, i.e., the queue backlogs remain finite over time. It is known that rate stability is a necessary condition for dynamic stability of the network [15].

It is quite easy to see that a necessary condition for rate stability is $r_{1}>r_{n}, n=2, \ldots, N$. Indeed, assuming that all the relay queues in the network are backlogged, they are rate stable if

$$
p \mathrm{e}^{-\lambda p c r_{1}^{2}}<p \mathrm{e}^{-\lambda p c r_{n}^{2}}, n=2, \ldots, N
$$

or, equivalently, $r_{1}>r_{n}, n=2, \ldots, N$. This condition can also be deduced by demanding that the denominators in (4) are greater than zero. We now propose a sufficient condition for rate stability.

Proposition 2: The relay queues are rate stable if $r_{1}>$ $\sqrt{N} r_{n}, n=2, \ldots, N$.

Proof: Since the sources are backlogged, the success probability in the first hop is the largest when no potential interfering relay has a packet to transmit. From (2), an upper bound to the success probability in the first hop is thus $\bar{p}_{1}^{s}=\mathrm{e}^{-\frac{\lambda}{N} p c r_{1}^{2}}$. Similarly, the success probability in the second hop is the smallest when all nodes that are potential interferers in the second hop have packets to transmit. In this case, the density of interferers is $\frac{N-1}{N} \lambda p+\frac{1}{N} \lambda p=\lambda p$ and a lower bound to the success probability in the second hop is $\underline{p}_{2}^{s}=\mathrm{e}^{-\lambda p c r_{2}^{2}}$. A sufficient condition for the stability of the first relay queue is therefore $\bar{p}_{1}^{s}<\underline{p}_{2}^{s}$. Defining $\underline{p}_{n}^{s}=\mathrm{e}^{-\lambda p c r_{n}^{2}}$ as the lower bound to the success probability of hop $n$, $n=2, \ldots, N$, a set of sufficient conditions for the stability of all relay queues is

$$
\bar{p}_{1}^{s}<\underline{p}_{n}^{s} \Leftrightarrow r_{1}>\sqrt{N} r_{n}, n=2, \ldots, N .
$$

The factor $\sqrt{N}$ is the result of the considered worst-case scenario, according to which the density of interferers in hop $n$ is $N$ times larger than the density of interferers in the first hop. Since packets arrive at the queues of the initially empty relays with positive probability, the packet arrival probability to the backlogged relays is expected to progressively decrease due to increasing interference. For this reason, it appears that Proposition 2 may be far from necessary. We conjecture that the condition $r_{1}>r_{n}, n=2, \ldots, N$, is also sufficient for the rate stability of the relay queues, which is corroborated by our simulation results in Section IV.

\section{NUMERICAL RESULTS}

In this section, we present analytical and simulation results with parameter values $\lambda=10^{-4}$ sources $/ \mathrm{m}^{2}, b=4$ and $p=0.05$. Since our analysis determines the mean delay over a typical route, in the simulation, the delay is evaluated as an average over time, over different routes, as well as over different network realizations. The simulated network size is $5000 \times 5000 \mathrm{~m}^{2}$, which on the average yields 2500 sources (and routes). In order to alleviate the impact of edge effects, metrics are collected only for routes with sources inside an inner square of size $1100 \times 1100 \mathrm{~m}^{2}$. The desired placement of the relays is determined by Proposition 1. Routing in each hop is performed by selecting the relay closest to and to the right of the respective desired point. As a result, routes with a smaller number of hops than that theoretically specified may exist in the network. The average end-to-end delay is calculated over those routes as well.

In Fig. 2, $D$ is plotted vs. $R$ for different numbers of hops. For each $N$, the distance of the first hop $r_{1}$ is determined such that (4) is minimized. Given $R$, an optimal number of hops exists that minimizes $D$. Moreover, the distance between the switching points (values of $R$ for which $N$ and $N+1$ hops yield the same delay) progressively increases, which points to a sublinear relationship between $N$ and $R$ (Fig. 6). Also note that the optimal total service time, plotted with a dashed line, provides a rather tight lower bound to the optimal end-to-end delay. This demonstrates that, for optimally placed relays and selected $N$, the total waiting time along the route is quite small compared to the service time.

The simulation results, shown with points, correspond to a relay density $\lambda_{r}=2(N-1) \lambda$. We observe that, in the range of $R$ for which a given number of hops is optimum, the match between the theoretical and simulated delay values is satisfactory. As $R$ increases, the average number of crossings between routes increases, so the discrepancy between theory and simulation becomes larger. We confirm that the discrepancy decreases with increasing relay density in Fig. 3. The 


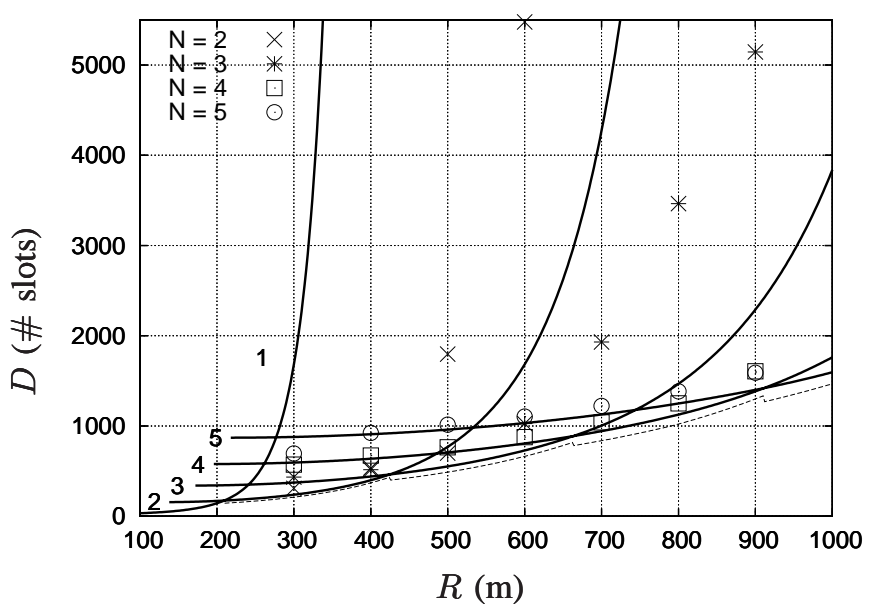

Fig. 2: $D$ vs. $R$, for $N=1, \ldots, 5$. The dashed line shows the optimal end-to-end service time. Simulation points are shown for $\lambda_{r}=2(N-1) \lambda$.

value $(N-1) \lambda$ is considered the minimum required relay density, as a route with $N$ hops employs $N-1$ relays.

In Fig. 4, we plot the simulated success probability in the first hop as a function of time, for a few randomly selected routes. The parameter values are $R=500 \mathrm{~m}, N=3$ and $\lambda_{r}=2 \lambda$. After a transient period, the time traces settle into their stationary values, which are close to, but smaller than the theoretical value $p_{1}^{s}=0.18$, computed by (6). This is due to the fact that the actual relays are situated close to but not exactly at the optimal positions.

In Fig. 5, we explore the sensitivity of the delay to the placement of the first relay, for three different pairs of values $(R, N)$. The curves correspond to the theoretical delay obtained by (4). The simulation confirms the validity of the analysis for a relay density $\lambda_{r}=2(N-1) \lambda$. The discrepancy at $r_{1}=450 \mathrm{~m}$ for $R=500 \mathrm{~m}$ is explained by the existence of a large number of two-hop routes in the network, for which the delay is very large, as the - only - relay is placed very close to the destination. Note that the delay is minimized if the distance of the first hop is significantly larger than the distance of the remaining hops, i.e., the optimal $r_{1}$ for $R=500 \mathrm{~m}(N=3)$ is approximately $250 \mathrm{~m}$, i.e., half the total distance, while the optimal $r_{1}$ for $R=1000 \mathrm{~m}(N=5)$ is $350 \mathrm{~m}$, i.e., about a third of the total distance. The presence of an optimal $r_{1}$ is explained as follows: when $r_{1} \rightarrow R, p_{2}^{s} \rightarrow 1$ and the service time at the source dominates the delay; on the other hand, as $r_{1} \rightarrow R / N$, i.e., all hops tend to become equidistant, $p_{2}^{s} \rightarrow p_{1}^{s}$ and the queueing delay at the relays obtains very large values, e.g., an $r_{1}$ approximately $100 \mathrm{~m}$ short of the optimal value results in a large delay penalty.

In Fig.6 we plot the optimal number of hops as a function of $R$. It is seen that this number scales sublinearly with $R$; specifically, we find that $N \approx \eta_{1} R^{0.74}$, where $\eta_{1}>0$ is determined by the sublinear fit. We can also verify that the optimal distance for the first hop also scales sublinearly with

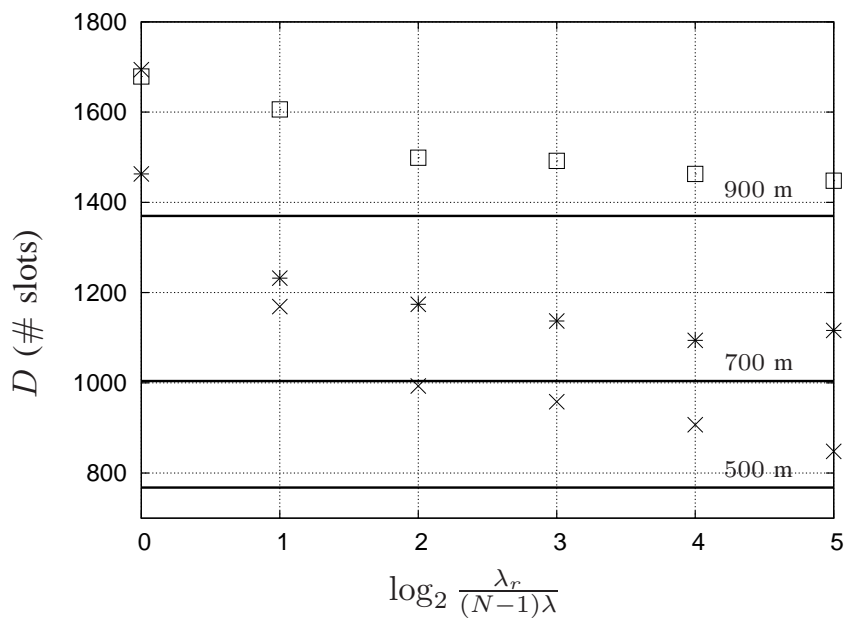

Fig. 3: $D$ vs. $\frac{\lambda_{r}}{(N-1) \lambda}$, for $R=500 \mathrm{~m}(N=2), R=700 \mathrm{~m}$ $(N=3)$ and $R=900 \mathrm{~m}(N=4)$.

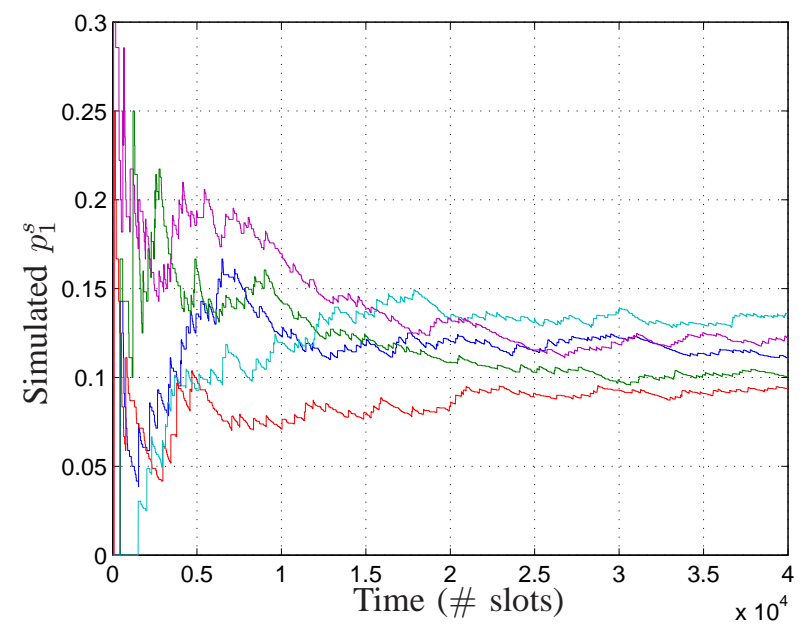

Fig. 4: Simulated $p_{1}^{s}$ vs. time, for five randomly selected routes $\left(R=500 \mathrm{~m}, N=3, \lambda_{r}=2 \lambda\right)$.

$R$, i.e., $r_{1} \approx \eta_{2} R^{0.43}, \eta_{2}>0$, and that as $R$ increases, $p_{2}^{s}$ approaches a constant value $\tilde{p}_{2}^{s} \approx 0.55$, while $p_{1}^{s}$ progressively decreases [13]. As a result, solving (6) over $p_{1}^{s}$ for large values of $R$, we have the following approximation

$$
p_{1}^{s} \approx \tilde{p}_{2}^{s} \frac{W\left(\frac{\lambda p c r_{1}^{2}}{\tilde{p}_{2}^{s}} \mathrm{e}^{-\frac{\lambda p c}{N} r_{1}^{2}}\right)}{\lambda p c r_{1}^{2}} .
$$

From Fig. 2, the optimal mean end-to-end delay can be approximated by the total service time so, for large $N$,

$$
D \approx \frac{\lambda c r_{1}^{2} N}{\tilde{p}_{2}^{s} W\left(\frac{\lambda p c r_{1}^{2}}{\tilde{p}_{2}^{s}} \mathrm{e}^{-\frac{\lambda p c}{N} r_{1}^{2}}\right)}+N^{2}\left(\frac{1}{p \tilde{p}_{2}^{s}}-1\right) .
$$

Taking into account that $p \ll 1$, we have

$$
D \approx \frac{\lambda c \eta_{1} \eta_{2}^{2} R^{1.6}}{\tilde{p}_{2}^{s} W\left(\frac{\eta_{3} \eta_{1}}{\tilde{p}_{2}^{s}} R^{0.74}\right)}+\frac{\eta_{1}^{2} R^{1.48}}{p \tilde{p}_{2}^{s}}
$$




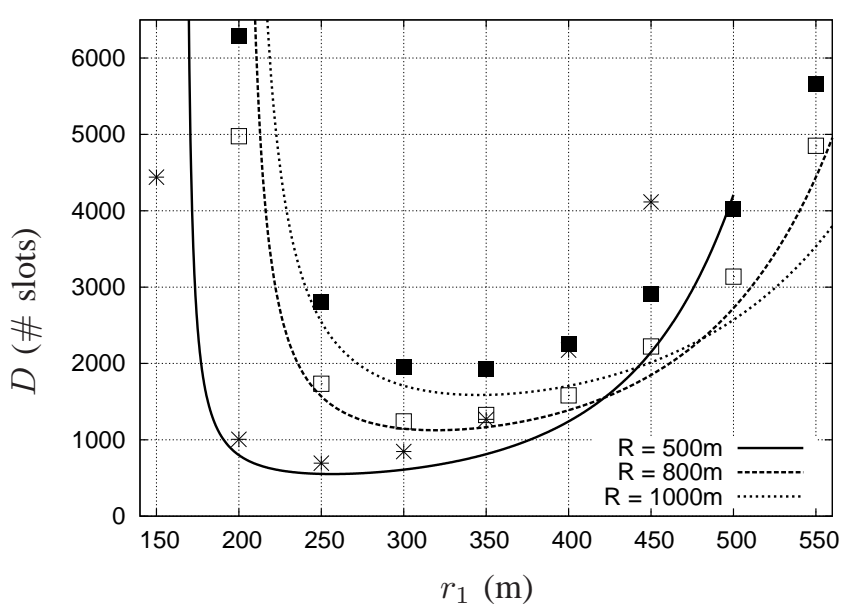

Fig. 5: $D$ vs. $r_{1}$, for $R=500,800,1000 \mathrm{~m}(N=3,4,5$ respectively). Simulation points are shown for $\lambda_{r}=2(N-$ 1) $\lambda$.

where $\eta_{3} \triangleq \frac{\lambda p c r_{1}^{2}}{N} \mathrm{e}^{-\frac{\lambda p c r_{1}^{2}}{N}} \leq \mathrm{e}^{-1}$. Since $W(x)=\mathcal{O}(\ln x)$ for $x \rightarrow \infty$, it also holds that $W(x)=\mathcal{O}(x)$. Moreover, $W(x)=\Omega(1)$. As a result, the first term in (10) is $\Omega\left(R^{0.86}\right)$ and $\mathcal{O}\left(R^{1.6}\right)$. The superlinear scaling of $D$ with $R$ in (10) is a result of the scaling of the optimal $r_{1}$ with $R$, as well as the TDMA nature of the MAC protocol, i.e., the constraint that a node is allowed to transmit only once every $N$ slots. These scaling results indicate that the MAC protocol is tailored to a small number of hops and, as $R$ increases, intra-route spatial reuse should be considered.

As a final side note, since $R$ is present in every expression in the product $\lambda c R^{2}$, for a given $R$, we also conclude that the optimal number of hops scales with $(\sqrt{\lambda c})^{0.74}$ or $(\lambda c)^{0.37}$.

\section{CONCLUDING REMARKS}

We conducted an analytical and simulation study of random, interference-limited, multi-hop networks. We first evaluated the mean end-to-end delay using an idealized network model and obtained the optimal placement of the relays and number of hops such that the delay is minimized. We then employed these design insights in order to perform routing in a realistic network environment, where the relays are picked from a random population. The simulation results confirmed that significant gains in terms of delay can be achieved, if routing is performed according to the optimal hopping distances indicated by the analysis. Moreover, we demonstrated that departure from these guidelines can lead to substantial delay penalties.

In conclusion, we view this work as a first step in understanding the end-to-end performance in random networks. Extending our analysis in order to accomodate varying sourcedestination distances, intra-route spatial reuse, or more complicated MAC/scheduling protocols, as well as providing a framework for a dynamical network analysis are all interesting topics for future research.

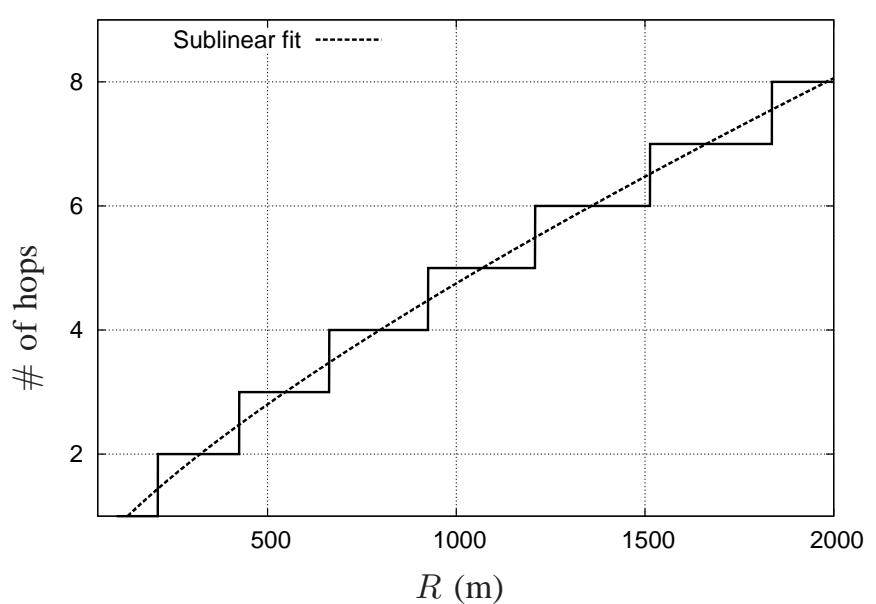

Fig. 6: Optimal number of hops vs. $R\left(\lambda=10^{-4}\right.$ sources $/ \mathrm{m}^{2}$, $p=0.05)$

\section{REFERENCES}

[1] M. Haenggi and D. Puccinelli, "Routing in ad hoc networks: a case for long hops," IEEE Commun. Mag., pp. 93-101, Oct. 2005.

[2] E. S. Sousa and J. A. Silvester, "Optimum transmission ranges in a direct-sequence spread-spectrum multi-hop packet radio network," IEEE J. Sel. Areas Commun., vol. 8, pp. 762-771, Jun. 1990.

[3] S. P. Weber, X. Yang, J. G. Andrews, and G. de Veciana, "Transmission capacity of wireless ad hoc networks with outage constraints," IEEE Trans. Inf. Theory, vol. 51, pp. 4091-4102, Dec. 2005.

[4] K. Stamatiou, J. G. Proakis, and J. R. Zeidler, "Evaluation of MIMO techniques in FH-MA ad hoc networks," in Proc. IEEE GLOBECOM, Washington D.C., Nov. 2007.

[5] F. Baccelli, B. Błaszczyszyn, and P. Mühlethaler, "An Aloha protocol for multi-hop mobile wireless networks," IEEE Trans. Inf. Theory, vol. 52, pp. 421-436, Feb. 2006.

[6] M. W. Subbarao and B. L. Hughes, "Optimal transmission ranges and code rates for frequency-hop packet radio networks," IEEE Trans. Commun., vol. 4, pp. 670-678, Apr. 2000.

[7] S. P. Weber, J. G. Andrews, and N. Jindal, "The effect of fading, channel inversion and threshold scheduling on ad hoc networks," IEEE Trans. Inf. Theory, vol. 53, pp. 4127-4149, Nov. 2007.

[8] M. Haenggi, "On routing in random Rayleigh fading networks," IEEE Trans. Wireless Commun., vol. 4, pp. 1553-1562, Jul. 2005.

[9] O. Oyman and S. Sandhu, "A Shannon theoretic perspective on fading multihop networks," in CISS 06, Princeton, Mar. 2006.

[10] M. Sikora, J. N. Laneman, M. Haenggi, D. J. Costello, and T. Fuja, "Bandwidth- and power-efficient routing in linear wireless networks," Joint Special Issue of IEEE Transactions on Information Theory and IEEE Transactions on Networking, vol. 52, pp. 2624-2633, Jun. 2006.

[11] F. Baccelli, B. Błaszczyszyn, and P. Mühlethaler, "On the performance of time-space opportunistic routing in multi-hop mobile ad hoc networks," in IEEE WiOpt 2008, Apr. 2008, pp. 307 - 316.

[12] A. Kumar, E. Altman, D. Miorandi, and M. Goyal, "New insights from a fixed-point analysis of single-cell IEEE 802.11 WLANs," IEEE/ACM Trans. Netw., vol. 15, pp. 588-601, Jun. 2007.

[13] K. Stamatiou, F. Rossetto, M. Haenggi, T. Javidi, J. R. Zeidler, and M. Zorzi, "Delay characterization of random multi-hop networks," 2009, to be submitted to the IEEE Transactions on Wireless Communications.

[14] R. K. Ganti and M. Haenggi, "Spatial and temporal correlation of the interference in ALOHA ad hoc networks," 2009, submitted to the IEEE Comm. Letters, available at http://arxiv.org/abs/0904.1444.

[15] M. Bramson, "Stability of queueing networks," Probability Surveys, vol. 5, pp. 169-345, Mar. 2008. 